\title{
Genetic Variability, Character Association and Path Coefficient Analysis of Yield Attributes for Medium and Late Maturing Potato Cultivars
}

\author{
Kaushik Kumar Panigrahi ${ }^{*}$, Jyotshnarani Pradhan², \\ Puranjaya Panigrahi ${ }^{3}$ and Kisor Kumar Sarkar ${ }^{3}$ \\ ${ }^{1}$ Department of Plant Breeding and Genetics, OUAT, Bhubaneswar, India \\ ${ }^{2}$ Department of Plant Physiology, Banaras Hindu University (BHU), Varanasi, India \\ ${ }^{3}$ Department of Genetics and Plant Breeding, Faculty of Agriculture, BCKV, West Bengal, India \\ *Corresponding author
}

\section{A B S T R A C T}

The present study emphasized on determination of the nature and magnitude of

Keywords

Correlation, K. (Kufri), C.V. (cultivated variety), GA, Heritability.

Article Info

Accepted:

23 June 2017

Available Online:

10 July 2017 variability, heritability and genetic advance on yield and yield attributing characters in 19 potato genotypes harvested on different date. The estimation of correlation co-efficient among the characters along with their direct and indirect effect on yield is necessary to carry out proper selection on the basis of the traits. Wide ranges of variation have been observed for all the traits both in early and late harvesting situation. High heritability was observed by dry matter \%, total tuber yield and marketable tuber yield for both the days of harvesting. Significant positive correlations were observed between total tuber yield with germination\%, non-marketable tuber yield and marketable tuber yield both at genotypic and phenotypic level at both 75 Days after planting and 90 days after planting. Most of the characters showed significant correlation among themselves in late harvest in compare to early. Genotypes like MS/1-4353, K. Pushkar, K. Surya, Chipsona-1, $\mathrm{K}$. Bahar and K. Pukhraj are very promising tuber yielder.

\section{Introduction}

Potato is an excellent low fat source of carbohydrates with one-fourth the calories of bread. When boiled it provides more protein than maize with nearly twice the calcium. Potato of an average size with skin provides about 10 percent of the recommended daily intake of fibre. Breeders should take the challenge to provide food at cheaper rate to the millions of hungry people in developing countries by increasing the production of potato per unit area and per unit time. To initiate any breeding programme to this direction, presence of enough genetic variability in the population for yield related traits should be considered as pre requisite element. Moreover, application of perfect breeding method is dependent on estimation of genetic gain of the characters for successful selection as to develop desirable traits suggested (Johnson et al., 1955). The estimation of correlation co-efficient among the characters is necessary to carry out proper selection on the basis of simultaneous selection of correlated characters. The effect of harvesting at different dates on tuber yield on different genotypes can identify high 
yielding varieties which can be harvested even earlier with satisfactory yield so that farmer get benefit by selling their produce at higher premium value in off-season and at the same time land will be free for next pre kharif crop. Therefore, the present study had emphasized the determination of the nature and magnitude of variability, heritability and genetic advance on different traits concerned with yield and effect of date of harvesting on yield and related traits.

\section{Materials and Methods}

The field experiment of the present study was carried out at the Block Seed Farm, Adisaptagram of Hooghly district in West Bengal, India. The experimental place was situated at $22.57^{\circ} \mathrm{N}$ latitude and $88.20^{\circ} \mathrm{E}$ longitudes with an elevation of 7.8 meters from mean sea level. The climate of this region is sub-tropical humid. And the entire year can be classified into three distinct seasons namely winter season, Summer season and Rainy Season. Occasionally late monsoon rain in the month of October causes some hazards to the planting of potato. The maximum and minimum temperature during the experiment ranged from $24.38-34.42$ and 9.51 - 25.05 respectively. During the investigation period the maximum mean monthly temperature was 35.92 in the month of March and the minimum mean monthly temperature was 12.54 in the month of December. The daily mean temperature remained congenial to sustain crop growth and tuberization up to the middle of February and thereafter the temperature began to shoot up. Very low rainfall occurred during the investigation period. The monthly maximum and minimum relative humidity $(\mathrm{RH})$ varied from $89.27-95.01 \%$ and $40.13-70.11 \%$ during the investigation period. The experiment was conducted in RBD Design with 19 genotypes in 3 replications.
The genotypes were harvested in two dates (75 days after planting and 90 days after planting). Cultivated variety K. Jyoti from farmer field taken as a local check variety for West Bengal condition because it's seems to be promising in West Bengal. For obtaining good harvest NPK was applied with a dose of 200: $150: 150 \mathrm{~kg} / \mathrm{ha}$ respectively. Intercultural operations and plant protection measures were taken from time to time and as and when required. Germination was recorded after 30 days of planting. Plant height was measured from ground level to top of the highest branch at the time of 50 days of crop growth. Five plants were selected from each plot and each replication for recording plant height. Marketable yield, Non-marketable yield, Harvest index, Total tuber Yield, Number of leaves/Plant, Leaf Area Index, Equatorial diameter, Polar diameter, Dry matter content were recorded.

\section{Results and Discussion}

The mean, range, phenotypic, genotypic and environmental variances, coefficient of variation $(\mathrm{CV})$, genotypic coefficient of variation (GCV), phenotypic coefficient of variation (PCV), heritability (broad sense), genetic advance (GA) and genetic advance as percentage of mean (GA as \% mean) of eleven characters from 19 genotypes which were harvested after 75 and 90 days after planting are presented in the (Tables 1 and 2).

Wide range of variation was observed for all the traits in early as well as late harvested genotypes. Total tuber yield, marketable tuber yield and non-marketable tuber yield showed satisfactory yield when harvested late. Randhawa and Kooner (1994) observed significantly higher yield when harvested late. There were significant differences in polar and equatorial diameter at 90 days of harvesting as compared to 75 days. Highest difference between PCV and GCV were 
observed for non-marketable tuber yield in early and late harvested potato genotypes. High magnitude of difference between PCV and GCV implied environment effect pre dominantly acting upon the expression of phenotypic behaviour of the character. High heritability was recorded in dry matter \% followed by germination \%, harvest index, total tuber yield and marketable tuber yield for both early and late harvested potato genotypes. Mishra et al., (2006) observed high GCV, heritability and GA with respect to plant height, total tuber yield, marketable tuber yield and dry matter content. Kim et al., (1993) observed more than $70 \%$ heritability for dry matter content in tuber for early and late harvested potato. High heritability coupled with high genetic advance in total tuber yield and marketable tuber yield was found indicating the influence of additive gene effect on these characters. Pandey et al., (2005) reported high GCV and PCV for marketable tuber yield, average tuber yield and total tuber yield/plot. Low heritability along with low GA in leaf/plant at 50 DAP revealed the influenced of interactions of several genes with little prospect for their inclusion in potato improvement programme. Bhagowati et al., (2002) observed low heritability for leaf/plant and tuber number.

\section{List of genotypes used in the experiment}

\begin{tabular}{|c|c|c|}
\hline Sl. No. & Genotypes & Source of Materials \\
\hline 1 & J/99-48 & Central Potato Research Institute, Kufri, Shimla \\
\hline 2 & J/99-242 & Central Potato Research Institute, Kufri, Shimla \\
\hline 3 & K. Ashoka & Central Potato Research Institute, Kufri, Shimla \\
\hline 4 & K. Chandramukhi & Central Potato Research Institute, Kufri, Shimla \\
\hline 5 & K. Khyati & Central Potato Research Institute, Kufri, Shimla \\
\hline 6 & $2001-P-26$ & Central Potato Research Institute, Kufri, Shimla \\
\hline 7 & MS/1-4353 & Central Potato Research Institute, Kufri, Shimla \\
\hline 8 & MS/1-4906 & Central Potato Research Institute, Kufri, Shimla \\
\hline 9 & K. Pushkar & Central Potato Research Institute, Kufri, Shimla \\
\hline 10 & K. Pukhraj & Central Potato Research Institute, Kufri, Shimla \\
\hline 11 & K. Bahar & Central Potato Research Institute, Kufri, Shimla \\
\hline 12 & K. Jyoti & AICRP on POTATO West Bengal Project, \\
\hline 13 & Chipsona-1 & Central Potato Research Institute, Kufri, Shimla \\
\hline 14 & Chipsona-2 & Central Potato Research Institute, Kufri, Shimla \\
\hline 15 & Chipsona-3 & Central Potato Research Institute, Kufri, Shimla \\
\hline 16 & MP/98-71 & Central Potato Research Institute, Kufri, Shimla \\
\hline 17 & K. Surya & Central Potato Research Institute, Kufri, Shimla \\
\hline 18 & K. Sindhuri & Central Potato Research Institute, Kufri, Shimla \\
\hline 19 & Atlantic & Central Potato Research Institute, Kufri, Shimla \\
\hline
\end{tabular}

K. - Kufri; Local check*- K. Jyoti 
Table.1 Variability and genetic parameters for different characters of potato genotypes harvested at 75 DAP

\begin{tabular}{|c|c|c|c|c|c|c|c|c|c|c|}
\hline \multirow{2}{*}{ Character } & \multirow{2}{*}{ Range } & \multirow{2}{*}{ Mean } & \multirow{2}{*}{$\mathrm{CD}$} & \multicolumn{3}{|c|}{ Variance } & \multirow{2}{*}{ GCV } & \multirow{2}{*}{ PCV } & \multirow{2}{*}{$h^{2}$} & \multirow{2}{*}{ GA } \\
\hline & & & & GV & PV & $\mathrm{EV}$ & & & & \\
\hline Germination $\%$ & $50.217-94.167$ & 76.875 & 13.922 & 140.986 & 211.654 & 70.668 & 15.4456 & 18.9247 & 0.6661 & 25.9684 \\
\hline Leaves no. at 50 DAP & $12.467-15.533$ & 13.798 & 2.188 & 0.500 & 2.245 & 1.746 & 5.1229 & 10.8569 & 0.2225 & 4.9783 \\
\hline $\begin{array}{l}\text { Plant Height at } 50 \text { DAP } \\
\text { (cm.) }\end{array}$ & $37.357-64.287$ & 48.954 & 6.372 & 49.116 & 63.920 & 14.804 & 14.3160 & 16.3316 & 0.7684 & 25.8513 \\
\hline Leaf Area Index & $0.187-0.337$ & 0.226 & 0.054 & 0.001 & 0.002 & 0.001 & 12.4278 & 19.1045 & 0.4232 & 16.6540 \\
\hline Harvest Index & $57.000-80.333$ & 66.860 & 11.986 & 22.865 & 75.244 & 52.378 & 7.1520 & 12.9739 & 0.3039 & 8.1217 \\
\hline Polar Diameter(mm.) & $39.767-67.217$ & 53.242 & 7.966 & 45.521 & 68.656 & 23.135 & 12.6722 & 15.5628 & 0.6630 & 21.2561 \\
\hline Equatorial Diameter(mm.) & $25.793-56.360$ & 43.399 & 7.815 & 55.890 & 78.160 & 22.270 & 17.2262 & 20.3712 & 0.7151 & 30.0076 \\
\hline Dry Matter \% & $14.350-23.370$ & 18.214 & 0.357 & 3.837 & 3.883 & 0.046 & 10.7545 & 10.8194 & 0.9880 & 22.0214 \\
\hline $\begin{array}{l}\text { Non Marketable tuber Yield } \\
\text { (in Kg.) }\end{array}$ & $0.267-3.267$ & 1.125 & 1.280 & 0.489 & 1.087 & 0.597 & 62.1441 & 92.6194 & 0.4502 & 85.8945 \\
\hline $\begin{array}{l}\text { Marketable Tuber Yield (in } \\
\text { Kg.) }\end{array}$ & $11.400-25.667$ & 18.652 & 2.473 & 14.521 & 16.751 & 2.230 & 20.4305 & 21.9431 & 0.8669 & 39.1855 \\
\hline Total Tuber Yield (in Kg.) & $11.900-28.033$ & 19.739 & 2.661 & 17.350 & 19.932 & 2.582 & 21.1022 & 22.6181 & 0.8704 & 40.5571 \\
\hline
\end{tabular}

Coefficient of variation (CV), genotypic coefficient of variation (GCV), phenotypic coefficient of variation (PCV), heritability (broad sense), genetic advance

(GA), Genotypic variance(GV), Phenotypic variance (PV), Environmental Variance (EV), Days after planting (DAP), Critical difference (CD).

Table.2 Variability and genetic parameters for different characters of potato genotypes harvested at 90 DAP

\begin{tabular}{|c|c|c|c|c|c|c|c|c|c|c|}
\hline \multirow{2}{*}{ Character } & \multirow{2}{*}{ Range } & \multirow{2}{*}{ Mean } & \multirow{2}{*}{$\mathrm{CD}$} & \multicolumn{3}{|c|}{ Variance } & \multirow{2}{*}{$\mathrm{GCV}$} & \multirow{2}{*}{ PCV } & \multirow{2}{*}{$h^{2}$} & \multirow{2}{*}{ GA } \\
\hline & & & & GV & PV & $\mathrm{EV}$ & & & & \\
\hline Germination \% & $43.967-97.330$ & 73.553 & 9.965 & 250.720 & 286.926 & 36.206 & 21.5274 & 23.0294 & 0.8738 & 41.4542 \\
\hline Leaves no. at 50 DAP & $12.200-15.933$ & 13.593 & 2.499 & 0.129 & 2.406 & 2.277 & 2.6461 & 11.4113 & 0.0538 & 1.2640 \\
\hline Plant Height at 50 DAP $(\mathrm{cm})$. & $33.650-66.890$ & 47.418 & 8.260 & 49.604 & 74.479 & 24.875 & 14.8529 & 18.1999 & 0.6660 & 24.9701 \\
\hline Leaf Area Index & $0.173-0.377$ & 0.219 & 0.076 & 0.002 & 0.004 & 0.002 & 21.8122 & 30.2497 & 0.5199 & 32.4001 \\
\hline Harvest Index & $60.333-89.333$ & 71.947 & 5.657 & 70.287 & 81.955 & 11.669 & 11.6526 & 12.5827 & 0.8576 & 22.2299 \\
\hline Polar Diameter(mm.) & $55.810-72.527$ & 66.121 & 9.143 & 14.462 & 44.940 & 30.478 & 5.7515 & 10.1386 & 0.3218 & 6.7212 \\
\hline Equatorial Diameter(mm.) & $48.957-67.797$ & 57.222 & 9.633 & 9.911 & 43.741 & 33.830 & 5.5018 & 11.5579 & 0.2266 & 5.3950 \\
\hline Dry Matter \% & $15.520-23.900$ & 19.580 & 0.385 & 5.654 & 5.708 & 0.054 & 12.1438 & 12.2017 & 0.9905 & 24.8975 \\
\hline $\begin{array}{l}\text { Non Marketable tuber Yield } \\
\text { (in Kg.) }\end{array}$ & $0.100-3.267$ & 1.168 & 1.621 & 0.338 & 1.269 & 0.958 & 49.7659 & 97.4248 & 0.2609 & 52.3676 \\
\hline $\begin{array}{l}\text { Marketable Tuber Yield (in } \\
\text { Kg.) }\end{array}$ & $14.467-27.333$ & 21.889 & 3.177 & 19.467 & 23.146 & 3.679 & 20.1565 & 21.9789 & 0.8410 & 38.0795 \\
\hline Total Tuber Yield (in Kg.) & $14.833-32.867$ & 23.054 & 3.206 & 22.005 & 25.753 & 3.748 & 20.3474 & 22.0120 & 0.8545 & 38.7459 \\
\hline
\end{tabular}

Coefficient of variation (CV), genotypic coefficient of variation (GCV), phenotypic coefficient of variation (PCV), heritability (broad sense), genetic advance

(GA), Genotypic variance(GV), Phenotypic variance (PV), Environmental Variance (EV), Days after planting (DAP), Critical difference (CD). 
Table.3 Genotypic $(\mathrm{G})$ and phenotypic $(\mathrm{P})$ correlation among different yield attributing characters in

Potato genotypes harvested at 75 DAP

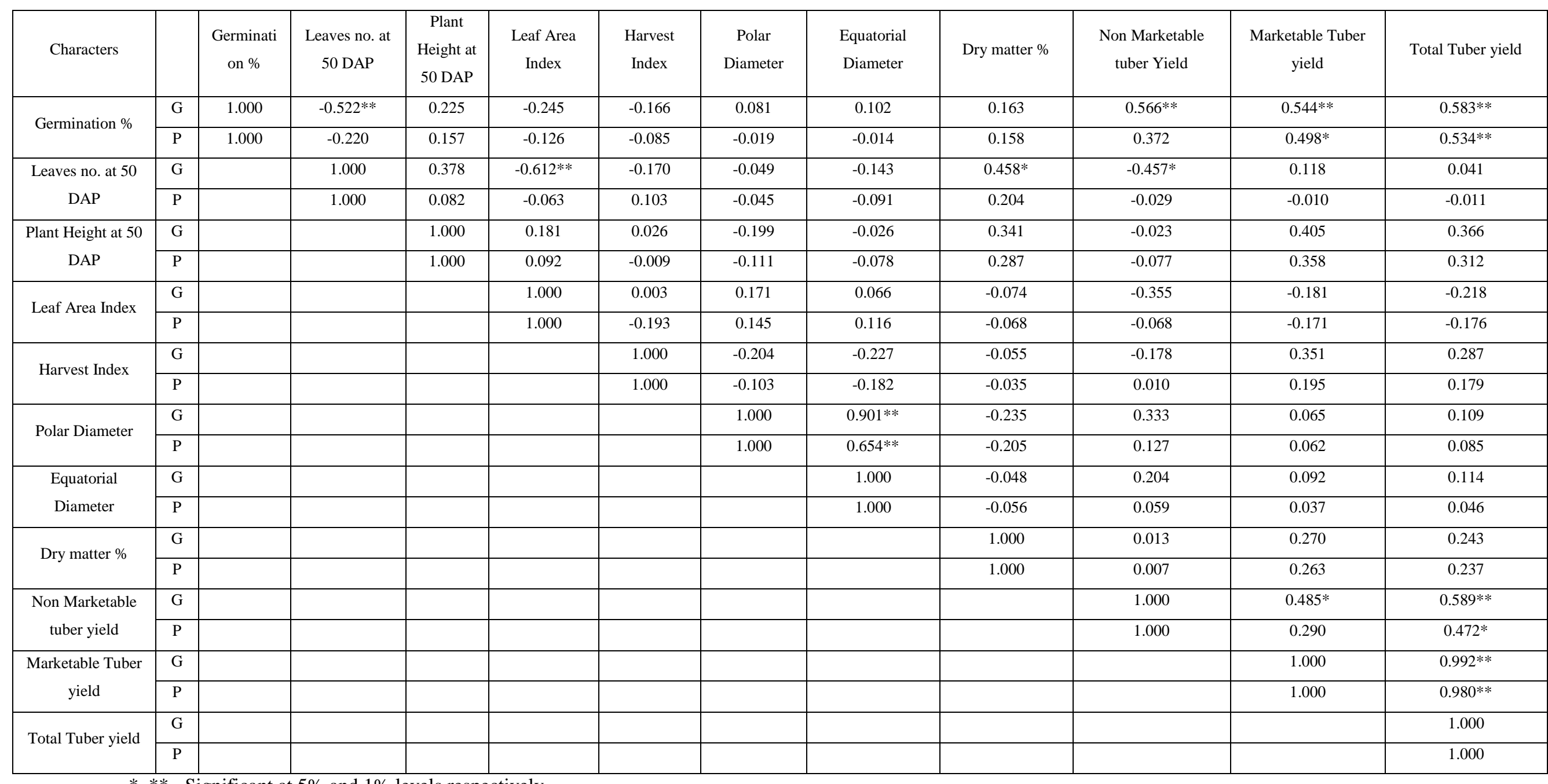

$*, * *=$ Significant at $5 \%$ and $1 \%$ levels respectively 
Table.4 Genotypic $(\mathrm{G})$ and phenotypic $(\mathrm{P})$ correlation among different yield attributing characters in Potato genotypes harvested at 90DAP

\begin{tabular}{|c|c|c|c|c|c|c|c|c|c|c|c|c|}
\hline Characters & & $\begin{array}{l}\text { Germin } \\
\text { ation \% }\end{array}$ & $\begin{array}{l}\text { Leaves no. } \\
\text { at } 50 \mathrm{DAP}\end{array}$ & $\begin{array}{c}\text { Plant } \\
\text { Height at } \\
50 \text { DAP }\end{array}$ & $\begin{array}{c}\text { Leaf Area } \\
\text { Index }\end{array}$ & $\begin{array}{l}\text { Harvest } \\
\text { Index }\end{array}$ & $\begin{array}{c}\text { Polar } \\
\text { Diameter }\end{array}$ & $\begin{array}{l}\text { Equatorial } \\
\text { Diameter }\end{array}$ & Dry matter $\%$ & $\begin{array}{c}\text { Non } \\
\text { Marketable } \\
\text { tuber Yield }\end{array}$ & $\begin{array}{l}\text { Marketable } \\
\text { Tuber yield }\end{array}$ & Total Tuber yield \\
\hline \multirow[t]{2}{*}{ Germination $\%$} & G & 1.000 & $-0.732 * *$ & $0.463^{*}$ & 0.142 & -0.272 & -0.039 & 0.222 & -0.264 & $0.948^{* * *}$ & 0.360 & $0.456^{*}$ \\
\hline & $\mathrm{P}$ & 1.000 & -0.098 & 0.397 & 0.120 & -0.224 & -0.041 & 0.188 & -0.252 & $0.457^{*}$ & 0.353 & 0.438 \\
\hline \multirow{2}{*}{$\begin{array}{c}\text { Leaves no. at } 50 \\
\text { DAP }\end{array}$} & G & & 1.000 & 0.298 & $-1.231 * *$ & 0.013 & $-0.641 * *$ & $-2.264 * *$ & $0.829 * *$ & $-2.290 * *$ & 0.105 & -0.190 \\
\hline & $\mathrm{P}$ & & 1.000 & 0.010 & -0.269 & 0.087 & -0.074 & -0.244 & 0.203 & -0.258 & 0.139 & 0.073 \\
\hline \multirow{2}{*}{$\begin{array}{c}\text { Plant Height at } 50 \\
\text { DAP }\end{array}$} & $\mathrm{G}$ & & & 1.000 & 0.053 & $-0.518 * *$ & 0.284 & 0.327 & -0.062 & 0.271 & 0.269 & 0.286 \\
\hline & $\mathrm{P}$ & & & 1.000 & 0.109 & -0.443 & 0.050 & 0.116 & -0.049 & 0.130 & 0.253 & 0.269 \\
\hline \multirow[t]{2}{*}{ Leaf Area Index } & G & & & & 1.000 & -0.352 & 0.034 & $1.060 * *$ & $-0.654 * *$ & $0.724 * *$ & -0.411 & -0.297 \\
\hline & $\mathrm{P}$ & & & & 1.000 & -0.263 & 0.012 & 0.268 & $-0.489 *$ & 0.370 & -0.261 & -0.163 \\
\hline \multirow[t]{2}{*}{ Harvest Index } & G & & & & & 1.000 & -0.058 & $-0.501 *$ & 0.266 & 0.004 & 0.315 & 0.296 \\
\hline & $\mathrm{P}$ & & & & & 1.000 & 0.005 & -0.149 & 0.236 & -0.019 & 0.309 & 0.289 \\
\hline \multirow[t]{2}{*}{ Polar Diameter } & $\mathrm{G}$ & & & & & & 1.000 & $0.518 * *$ & -0.040 & $0.588^{* * *}$ & 0.184 & 0.247 \\
\hline & $\mathrm{P}$ & & & & & & 1.000 & 0.408 & -0.034 & -0.184 & 0.074 & 0.029 \\
\hline \multirow{2}{*}{$\begin{array}{l}\text { Equatorial } \\
\text { Diameter }\end{array}$} & G & & & & & & & 1.000 & $-0.672 * *$ & $0.939^{* *}$ & -0.393 & -0.253 \\
\hline & $\mathrm{P}$ & & & & & & & 1.000 & -0.347 & 0.013 & -0.094 & -0.085 \\
\hline \multirow[t]{2}{*}{ Dry matter $\%$} & G & & & & & & & & 1.000 & -0.399 & 0.397 & 0.324 \\
\hline & $\mathrm{P}$ & & & & & & & & 1.000 & -0.183 & 0.358 & 0.298 \\
\hline \multirow{2}{*}{$\begin{array}{c}\text { Non Marketable } \\
\text { tuber Yield }\end{array}$} & G & & & & & & & & & 1.000 & 0.428 & $0.527 * *$ \\
\hline & $\mathrm{P}$ & & & & & & & & & 1.000 & 0.120 & 0.339 \\
\hline \multirow{2}{*}{$\begin{array}{c}\text { Marketable Tuber } \\
\text { yield }\end{array}$} & G & & & & & & & & & & 1.000 & $0.994 * *$ \\
\hline & $\mathrm{P}$ & & & & & & & & & & 1.000 & $0.975^{* *}$ \\
\hline \multirow[t]{2}{*}{ Total Tuber yield } & G & & & & & & & & & & & 1.000 \\
\hline & $\mathrm{P}$ & & & & & & & & & & & 1.000 \\
\hline
\end{tabular}


Table.5 Path coefficient analysis for potato genotypes harvested at 75 DAP

\begin{tabular}{|c|c|c|c|c|c|c|c|c|c|c|c|}
\hline Characters & $\begin{array}{c}\text { Germinati } \\
\text { on \% }\end{array}$ & $\begin{array}{c}\text { Leaves } \\
\text { n. at 50 } \\
\text { DAP }\end{array}$ & $\begin{array}{c}\text { Plant } \\
\text { Height at }\end{array}$ & $\begin{array}{c}\text { Leaf Area } \\
\text { Index }\end{array}$ & $\begin{array}{c}\text { Harvest } \\
\text { Index }\end{array}$ & $\begin{array}{c}\text { Polar } \\
\text { Diameter }\end{array}$ & $\begin{array}{c}\text { Equatorial } \\
\text { Diameter }\end{array}$ & $\begin{array}{c}\text { Dry matter } \\
\%\end{array}$ & $\begin{array}{c}\text { Non } \\
\text { Marketable } \\
\text { tuber Yield }\end{array}$ & $\begin{array}{c}\text { Marketable } \\
\text { Tuber yield }\end{array}$ & $\begin{array}{c}\text { Genotypic } \\
\text { yield } \\
\text { correlation }\end{array}$ \\
\hline Germination \% & $\mathbf{- 0 . 0 0 1 8 3}$ & -0.00219 & -0.00317 & -0.00187 & 0.00375 & -0.00153 & 0.00105 & -0.00246 & 0.07693 & 0.51452 & $0.583 * *$ \\
\hline Leaves no. at 50 DAP & 0.00096 & $\mathbf{0 . 0 0 4 2 0}$ & -0.00532 & -0.00468 & 0.00384 & 0.00092 & -0.00147 & -0.00692 & -0.06220 & 0.11158 & 0.041 \\
\hline $\begin{array}{c}\text { Plant Height at 50 } \\
\text { DAP }\end{array}$ & -0.00041 & 0.00159 & $\mathbf{- 0 . 0 1 4 0 7}$ & 0.00139 & -0.00060 & 0.00375 & -0.00027 & -0.00516 & -0.00318 & 0.38267 & 0.366 \\
\hline Leaf Area Index & 0.00045 & -0.00257 & -0.00255 & $\mathbf{0 . 0 0 7 6 4}$ & -0.00007 & -0.00323 & 0.00068 & 0.00113 & -0.04834 & -0.17133 & -0.218 \\
\hline Harvest Index & 0.00030 & -0.00071 & -0.00037 & 0.00003 & $\mathbf{- 0 . 0 2 2 5 9}$ & 0.00385 & -0.00233 & 0.00083 & -0.02423 & 0.33185 & 0.287 \\
\hline Polar Diameter & -0.00015 & -0.00021 & 0.00280 & 0.00131 & 0.00461 & $\mathbf{- 0 . 0 1 8 8 3}$ & 0.00928 & 0.00355 & 0.04522 & 0.06110 & 0.109 \\
\hline Equatorial Diameter & -0.00019 & -0.00060 & 0.00037 & 0.00050 & 0.00512 & -0.01696 & $\mathbf{0 . 0 1 0 2 9}$ & 0.00072 & 0.02779 & 0.08692 & 0.114 \\
\hline Dry matter \% & -0.00030 & 0.00192 & -0.00480 & -0.00057 & 0.00125 & 0.00443 & -0.00049 & $\mathbf{- 0 . 0 1 5 1 1}$ & 0.00173 & 0.25513 & 0.243 \\
\hline $\begin{array}{c}\text { Non Marketable tuber } \\
\text { Yield }\end{array}$ & -0.00104 & -0.00192 & 0.00033 & -0.00272 & 0.00402 & -0.00626 & 0.00210 & -0.00019 & $\mathbf{0 . 1 3 5 9 8}$ & 0.45868 & $0.589 * *$ \\
\hline $\begin{array}{c}\text { Marketable Tuber } \\
\text { yield }\end{array}$ & -0.00100 & 0.00049 & -0.00569 & -0.00138 & -0.00792 & -0.00122 & 0.00095 & -0.00408 & 0.06593 & $\mathbf{0 . 9 4 5 9 4}$ & 0.992 \\
\hline
\end{tabular}
Residual Effect $=0.6802012, *, * *=$ Significant at $5 \%$ and $1 \%$ levels respectively

Table.6 Path coefficient analysis for potato genotypes harvested at 90 DAP

\begin{tabular}{|c|c|c|c|c|c|c|c|c|c|c|c|}
\hline Characters & $\begin{array}{c}\text { Germinatio } \\
\mathrm{n} \%\end{array}$ & $\begin{array}{l}\text { Leaves no. } \\
\text { at } 50 \text { DAP }\end{array}$ & $\begin{array}{c}\text { Plant } \\
\text { Height at } \\
\text { 50 DAP }\end{array}$ & $\begin{array}{l}\text { Leaf Area } \\
\text { Index }\end{array}$ & $\begin{array}{l}\text { Harvest } \\
\text { Index }\end{array}$ & $\begin{array}{c}\text { Polar } \\
\text { Diameter }\end{array}$ & $\begin{array}{l}\text { Equatorial } \\
\text { Diameter }\end{array}$ & $\begin{array}{c}\text { Dry matter } \\
\%\end{array}$ & $\begin{array}{c}\text { Non } \\
\text { Marketable } \\
\text { tuber Yield }\end{array}$ & $\begin{array}{l}\text { Marketable } \\
\text { Tuber yield }\end{array}$ & $\begin{array}{l}\text { Genotypic yield } \\
\text { correlation }\end{array}$ \\
\hline Germination $\%$ & -0.00300 & 0.00048 & -0.00086 & -0.00081 & 0.00073 & 0.00002 & 0.00082 & 0.00016 & 0.11945 & 0.33918 & $0.456^{*}$ \\
\hline $\begin{array}{c}\text { Leaves no. at } 50 \\
\text { DAP }\end{array}$ & 0.00220 & -0.00065 & -0.00056 & 0.00698 & -0.00003 & 0.00040 & -0.00835 & -0.00051 & -0.28845 & 0.09887 & -0.190 \\
\hline $\begin{array}{c}\text { Plant Height at } \\
50 \text { DAP }\end{array}$ & -0.00139 & -0.00019 & -0.00187 & -0.00030 & 0.00139 & -0.00018 & 0.00121 & 0.00004 & 0.03420 & 0.25353 & 0.286 \\
\hline Leaf Area Index & -0.00043 & 0.00080 & -0.00010 & -0.00567 & 0.00095 & -0.00019 & 0.00391 & 0.00040 & 0.09117 & -0.38747 & -0.297 \\
\hline Harvest Index & 0.00082 & -0.00001 & 0.00097 & 0.00200 & -0.00269 & 0.00004 & -0.00185 & -0.00016 & 0.00057 & 0.29621 & 0.296 \\
\hline Polar Diameter & 0.00012 & 0.00042 & -0.00053 & -0.00172 & 0.00016 & -0.00062 & 0.00191 & 0.00002 & 0.07403 & 0.17330 & 0.247 \\
\hline $\begin{array}{c}\text { Equatorial } \\
\text { Diameter }\end{array}$ & -0.00067 & 0.00148 & -0.00061 & -0.00601 & 0.00135 & -0.00032 & 0.00369 & 0.00041 & 0.11835 & -0.37026 & -0.253 \\
\hline Dry matter $\%$ & 0.00079 & -0.00054 & 0.00012 & 0.00371 & -0.00072 & 0.00003 & -0.00248 & -0.00061 & -0.05021 & 0.37365 & 0.324 \\
\hline $\begin{array}{c}\text { Non Marketable } \\
\text { tuber Yield }\end{array}$ & -0.00285 & 0.00149 & -0.00051 & -0.00410 & -0.00001 & -0.00036 & 0.00346 & 0.00024 & 0.12599 & 0.40320 & $0.527 * *$ \\
\hline $\begin{array}{l}\text { Marketable } \\
\text { Tuber yield }\end{array}$ & -0.00108 & -0.00007 & -0.00050 & 0.00233 & -0.00085 & -0.00011 & -0.00145 & -0.00024 & 0.05394 & 0.94176 & $0.994 * *$ \\
\hline
\end{tabular}
Residual Effect $=0.692551, *, * *=$ Significant at $5 \%$ and $1 \%$ levels respectively 
Total tuber yield at early harvest showed significant positive correlation with germination \%, marketable tuber yield and non-marketable tuber yield both at genotypic and phenotypic levels (Table 3). Similarly germination also showed significant positive association with marketable as well as nonmarketable tuber yield. Polar and equatorial diameter of tubers showed significant positive associations among themselves at phenotypic as well as genotypic levels. Dry matter \% showed significant positive association with leaves/plant at 50 DAP. Total tuber yield at late harvested potato (Table 4) showed positive and significant correlation with marketable tuber yield at both phenotypic as well as at genotypic levels. Birhman and Kaul (1989) showed high correlation with total tuber yield and marketable tuber yield. Similarly total tuber yield exhibited significant positive association with germination percentage and non-marketable tuber yield when harvested late. Leaf area index showed negative though not significant association with non-marketable tuber yield in early harvested potato. On the contrary it showed significant positive association at genotypic level when harvested late. Geamanu and Birnaure (1989) showed positive correlation between non marketable tuber yield and leaf area index.

At early harvest (Table 5) highest direct effect on total tuber yield was exerted by total marketable tuber yield followed by nonmarketable tuber yield. Roy and Singh (2006) observed plant height, no. of tubers/plant and marketable yield to exert positive direct effect on total yield. Direct effects of other traits were found to be negligible. Germination percentage showed negative direct effect on total tuber yield contributed mainly via indirect effect of marketable tuber yield although strong positive association was observed between total tuber yield and germination percentage. Kim et al., (1993) showed different pattern of direct and indirect effect of characters due to change of date of harvesting. For late harvested genotypes (Table 6) highest direct effect on total tuber yield was exerted by total marketable yield followed by non-marketable tuber yield. Pandey et al., (2005) observed positive direct effect of marketable tuber yield and tuber/plant with total tuber yield. Strong positive association was shown between total tuber yield and germination percentage and it's influenced on the trait was mainly influenced by indirect effect via marketable tuber yield.

\section{References}

Bhagowati, R.R. Saikia, M. and Sut, D. 2002. Variability, heritability, genetic advance and character association in True Potato Seed (TPS) populations. Journal of the Agricultural Science Society of North East India, 15 (1): 119-122

Birhman, R. K. and Kaul, M.L.H. 1989. Variability components and genetic parameters in Andigena potato Biologisches Zentralblatt, 108 (3): 209219

Johnson, H.W. Robinson, H.F. and Comstock, R.E. 1955. Estimates of genetic and environmental variability in soybeans. Agron J 47: 314-318

Kim, J.K. Cho, H.M. Kim, S.Y. and Cho, C.H. 1993. Early and late varietal differences on growth, development, dry matter accumulation patterns and genetic characters of potatoes (Solanum tuberosum L.) in alpine areas. $R D A$ Journal of Agricultural Science Horticulture. 35 (2): 442-451

Mishra, A.C. Singh, N.P. Shashi Kamal. and Virender Kumar. 2006. Studies on genetic variability, heritability and genetic advance in potato (Solanum tuberosum L.).International Journal of 
Plant Sciences Muzaffarnagar. 1(1): 3941

Pandey, S.K. Singh, S.V. and Manivel, P. 2005. Genetic variability and causal relationship over seasons in potato. Crop Research Hisar. 29 (2): 277-281

Pandey, S. K. Singh, S.V. and Manivel, P. 2005. Yield structure, agronomic performance and stability of new potato (Solanum tuberosum) hybrids in western Uttar Pradesh. Indian Journal of Agricultural Sciences.75 (7): 417-421
Randhawa, K.S. and Kooner, K.S. 1994. Evaluation of new cultivars of potato (Solanum tuberosum L.) in relation to time of sowing and harvesting. Acta Horticulturae. 37 (1): 227-233

Roy, A.K. and Singh, P.K. 2006. Genetic variability, heritability and genetic advance for yield in potato (Solanum tuberosum L.). International Journal of Plant Sciences Muzaffarnagar. 1 (2): 282-285.

\section{How to cite this article:}

Kaushik Kumar Panigrahi, Jyotshnarani Pradhan, Puranjaya Panigrahi and Kisor Kumar Sarkar. 2017. Genetic Variability, Character Association and Path Coefficient Analysis of Yield Attributes for Medium and Late Maturing Potato Cultivars. Int.J.Curr.Microbiol.App.Sci. 6(7): 2558-2566. doi: https://doi.org/10.20546/ijcmas.2017.607.302 